Mens

revue d'histoire intellectuelle de l'Amérique française

\title{
III. Une vie intellectuelle active : l'antagonisme libéral-ultramontain (1840-1880)
}

Volume 6, numéro supplément, 2005-2006

URI : https://id.erudit.org/iderudit/1024238ar

DOI : https://doi.org/10.7202/1024238ar

Aller au sommaire du numéro

Éditeur(s)

Centre de recherche en civilisation canadienne-française

ISSN

1492-8647 (imprimé)

1927-9299 (numérique)

Découvrir la revue

Citer ce document

(2005). III. Une vie intellectuelle active : l'antagonisme libéral-ultramontain (1840-1880). Mens, 6, 28-41. https://doi.org/10.7202/1024238ar d'utilisation que vous pouvez consulter en ligne. 
WATSON, Samuel, "United States Army Officers Fight the "Patriot War": Responses to Filibustering on the Canadian Border, 1837-1839 ", Journal of the Early Republic, 18, 3 (automne 1998), p. 485-519.

YOUNG, Brian, "The Volunteer Militia in Lower Canada (18371850) ", dans Poner, Place and Identity: Historical Studies of Social and Legal Regulation in Quebec, aites d'un colloque du Groupe sur l'bistoire de Montréal (1996), Montréal, The Group, 1998, p. 37-53.

\section{Une vie intellectuelle active : l'antagonisme libéral- ultramontain (1840-1880)}

BALMER, Randall et Catharine Randall, " "Her Duty to Canada": Henriette Feller and French Protestantism in Quebec », Churis History, 70, 1 (2001), p. 49-72.

BARRIÈRE, Mireille, «Le goupillon, le maillet et la censure du théâtre lyrique à Montréal (1840-1914) ", Les Cahiers des Dix, 54 (2000), p. 119-135.

BEAUDOIN, Réjean, « Réception critique de la littérature québécoise au Canada anglais (1867-1901) », Études françaises, 32, 3 (1996), p. 61-76.

BÉLAND, Cindy, «Salons et soirées mondaines au Canada français : un espace privé vers l'espace public », dans Pierre Rajotte (dir.), Lieux et réseaux de sociabilité littéraire au Québec, Québec, Éditions Nota bene, 2001, p. 71-112.

BÉLISLE, Jean, «Une résidence oubliée : la maison de LouisHyppolite La Fontaine ", Journal of Canadian Art History/Annales d'histoire de l'art canadien, 20, 1-2 (1999), p. 46-67. 
BÉLISLE, Jean-François, « Garcì M Ioreno de carne y hueso ou le caractère mythique du modèle d'homme d'État chrétien dans les milieux ultramontains du Canada français ", dans Jean-Pierre Wallot (dir.), avec la collaboration de Pierre Lanthier et Hubert Watelet, Constructions identitaires et pratiques sociales sociales : actes $d u$ colloque en hommage à Pierre Savard, tenu à l'Université d'Ottawa les 4, 5, 6 octobre 2000, Ottawa, Les Presses de l'Université d'Ottawa, 2002, p. 344-360.

BENARDI, Roberto. Le royage au Canada français et en Amérique du Nord : exotisme et modernité dans la France de la seconde moitié du XIXe siècle, Ph.D. (Études françaises), Université de Montréal, 1999, 294 p.

BERREHAR, Marie-Hélène, François-Xaicer Garneau et Jules Michelet : figures du peuple, Montréal, Centre d'études québécoises, Département d'études françaises, Université de Montréal, 1997, $128 \mathrm{p}$.

BIENVENUE, Louise, «Pierres grises et mauvaise conscience : essai historiographique sur le rôle de l'Église catholique dans l'assistance au Québec ", Études d'histoire religieuse/Historical Studies. The Canadian Catholic Historical Association, 69 (2003), p. 9-28.

BILODEAU, Maryse, La diffusion par les imprimés des connaissances sur les plantes médicinales au Québec de 1860 à 1950, M.A. (Histoire), Université de Sherbrooke, 1999, 162 p.

BOUCHARD, Chantal, La langue et le nombril. Histoire d'une obsession québéioise, Montréal, Fides, 1998, 303 p.

BOUCHARD, Gérard, Bernard CASGRAIN, Mario BOURQUE et Raymond ROY, "Le fichier de population BALSAC. Situation et perspectives ». Annales de démographie historique, 2, 1998, p. 187-196.

BOUCHARD, Gérard, "Through the Meshes of Patriarchy: the Male/Female Relationship in the Saguenay Peasant Society (18601930) ». The History of the Family. An International Quarterly, 4, 4, 2000 , p. 397-425. 
BOUCHARD, Gérard, «La sexualité comme pratique et rapport social chez les couples paysans du Saguenay (1860-1930) ». Reine d'histoire de l'Amérique française, 54, 2 (automne) 2000, p. 183-217.

BOUCHARD, Gérard, «L'évolution économique et socioprofessionnelle de la structure des emplois dans la région du Saguenay (1842-1971) ", dans A.-L. Head-König, L. Lorenzetti, B. Veyrassat (éds.), Famille, parenté et réseaù en Ocicident (XI TIe-XXe siècles). Mélanges offerts à Alfred Perrenoud. Genève, Société d'Histoire et d'Archéologie de Genève, 2001, p. 293-327.

BROSSEAU, Marc, "L'architecture de la géographie scolaire québécoise (1840-1960) ", Cahiers de géographie du Québec, 43, 120 (décembre 1999), p. 561-584.

CAMPAGNA, Christine, Le rôle de la presse i'u par les propriétaires et les rédacteurs de journaux montréalais, 1830-1880, M.A. (Histoire), Université du Québec à Montréal, 1998, 144 p.

CHALIFOUX, Éric, Entre le gouternement des hommes et celui des âmes : le rapport au politique dans les discours ultramontain et libéral québécois, 1860-1880, M.A. (Histoire), Université du Québec à Montréal, 1998, 107 p.

CHARLAND, Jean-Pierre, L'entreprise éducatice au Québec (18401900), Sainte-Foy, Les Presses de l'Université Laval, 2000, 452 p.

CHEVRIER, Marc, « Au royaume de la néomonarchie », Liberté, 40 (avril 1998), p. 75-88.

CHEVRIER, Marc, " De la monarchie en Amérique », L'Action nationale, LXXXVII, 5 (mai 1998), p. 87-129.

CLERK, Bruno, Le joumal The True Witness and Catholic Chronicle et la pensée religieuse de George Edn'ard Clerk (1850-1875), M.A. (Histoire), Université de Montréal, 1996, 179 p.

CLICHE, Narie-Aimée, " "Qui bene amat bene castignat" : Le débat pédagogique sur les punitions corporelles dans les revues pédagogiques du Québec, 1857- 1964 », Historical Studies in Education/Rerue d'bistoire de l'éducation, 11, 2, (automne 1999), p. 147-169. 
COLIN, Johanne et Laurence MONNAIS-ROUSSELOT, «La communauté médicale montréalaise de 1850 à 1890 : variations sur le thème d'élite », Histoire sociale/Social History, 32, (novembre 1999), p. 173-208.

COLLIN, Marc, Mensonges et vérités dans les Soucenirs de Félix Poutré : essai d'analyse psychohistorique d'un cas de fraude historique, M.A. (Histoire), Université du Québec à Montréal, 2002, 176 p.

COLLIN, Jean-Pierre et Michèle DAGENAIS, «Évolution des enjeux politiques locaux et des pratiques municipales dans l'île de Montréal (1840-1950)", dans Denis Menjot et Jean-Luc Pinol (dirs.), Enjeux et expressions de la politique municipale (XIIe-XXe siècles), Paris, L'Harmattan, 1997, p. 191-221.

CORBETT, Nike, «A Protracted Struggle: Rural Resistance and Normalization in Canadian Education History ", Historical Studies in Education/Rerue d'bistoire de l'éducation, 13, 1, (printemps 2001), p.147-169.

COUPAL, Éric, Baseball, américanité et culture populaire : histoire du baseball à Montréal 1860-1914, M.A. (Histoire), Université du Québec à Montréal, 2001, v-109 p.

COURVILLE, Serge, Immigration, colonisation et propagande : du rêie américain au rềe colonial, Sainte-Foy, Éditions Multi-M Iondes, 2002, $700 \mathrm{p}$.

DAVID, Éric, Le clergé catholique canadien-français et la théorie de l'érolution (1860-1950), M.A. (Histoire), Université du Québec à Montréal, 2003, 99 p.

DE SÈVE, Michel et Gérard BOUCHARD, «Long-term Intergenerational Mobility in Québec (1851-1951): the Emergence of a New Social Fluidity Regime ", Canadian Joumal of Sociology, 23, 4 (automne), 1998, p. 349-367.

DE SÈVE, Michel, Gérard BOUCHARD et Martin HAMEL, "Un siècle de mobilité professionnelle : un aperçu régional », Recherches sociographiques, XL, 1, 1999, p. 55-81. 
DÉRY, Boris, L a librairie Rolland (1842-1888), M.A. (Histoire), Université du Québec à Montréal, 2003, ix-188p.

DESHARNAIS, Josée, La gestion des loisirs publics à Montréal: l'exemple du parc de l'ille Sainte-Hélène (1874-1914), M.A. (Histoire), Université de Montréal, 1998, 119 p.

DESSAULLES, Louis-Antoine, Discours sur la tolérance, suili $d u$ mémoire de l'éléque Bourget, Présentation et notes par Adrien Thério, XYZ éditeur, coll. « Documents », 2002, 103 p.

FABRE, Gérard, «Prémisses de la pensée économique au Canada français : l'ambiguité de l'influence française ", dans Pierre Dockès et al. (dirs.), Les traditions éionomiques françaises (1848-1939), Paris, CNRS Éditions, 2000, p. 955-965.

FAHMY-EID, Nadia, "L'histoire de l'éducation des filles : bilan et perspective d'avenir ", Historical Studies in Education/Reine d'histoire de l'éducation, 15, 1 (printemps 2003), p. 1-17.

FECTEAU, Jean-Marie, Sylvie MÉNARD, Véronique STRINELLE et Jean TRÉPANIER, «Une politique de l'enfance délinquante et un danger : la mise en place des écoles de réforme et d'industrie au Québec (1840-1873) ", Crime, Histoire et Société, 2, 1 (1998), p. 75-110.

FOR'TIN, Philippe, Le journal Le Pays et la guerre de Sécession (18611865), M.A. (Histoire), Université du Québec à Montréal, 2000, $102 \mathrm{p}$.

FORTIN, Philippe, "La guerre de Sécession dans le journal Le Pays (1861-1865) ", Cahiers d'histoire, 21, 1 (automne 2001) , p. 94114.

FORTIN, Philippe, "Les sources de renseignement du journal Le Pays lors de la guerre de Sécession (1861-1865) ", Communication, 20, 2 (hiver-printemps 2001), p. 118-131.

GAGNON, Serge, Religion, moralité et modernité, Sainte-Foy, Les Presses de l'Université Laval, 1999, 191 p. 
GALARNEAU, Claude, "Clergé, bourgeoisie et lecture publique. La bibliothèque paroissiale de Notre-Dame de Québec (18421847), Les Cahiers des Dix; 54 (2000), p. 99-117.

GALLILAND, Jason A., Redimensioning Montreal: Circulation and Urban Form (1846-1918), Ph.D. (Géographie), Université McGill, 2001, xiii-277 p.

GAUTHIER-LAROUCHE, Georges, "Le voyage d'HenriDavid Thoreau au Canada en 1850 », dans Jean-Pierre Pichette (dir.), Entre Beauce et Acadie : facettes d'un parcours ethnologique. Études offertes au professeur Jean-Claude Dupont, Sainte-Foy, Les Presses de l'Université Laval, 2001, p. 374-380.

GILBERT, Anne, "À propos du concept d'Amérique française ", Recherches sociographiques, 39, 1 (janvier-avril 1998), p. 103-120.

GRACE, Robert John, The Irish in Mid-Nineteenth-Century Canada and the Case of Quebec: Immigration and Settlement in a Catholic City, Ph.D. (Histoire), Université Laval, 1999, 2 vol, 660 [39] p.

GRACE, Robert J., "Irish Immigration and Settlement in a Catholic City: Quebec, 1842-1861 ", The Canadian Historical Retien, 84, 2 (juin 2003), p. 217-251.

GROULX, Patrice, "La commémoration de la bataille de SainteFoy : du discours de la loyauté à la "fusion des races" ", Reine d'histoire de l'Amérique française, 55, 1 (été 2001), p. 45-83.

HAMELIN, Charles, Daniel Coussirat (1841-1907): la vie et l'aurre d'un intellectuel franio-protestant, M.A. (Histoire), Université de Montréal, 2001, $260 \mathrm{p}$.

HARDY, René, « Les conceptions prénuptiales à Trois-Rivières comme indice de fidélité religieuse (1850-1945)", Rel'ue d'histoire de l'Amérique française, 54, 4 (printemps 2001), p. 531-555.

HARVEY, Fernand, «Le diocèse catholique au Québec : un cadre territorial pour l'histoire sociale ", Les Cahiers des Dix, 56 (2002), p. 51-124. 
HÉBER'T, Yves, « Sir Adolphe-Basile Routhier », Dictionnaire biographique du Canada, XIV (de 1911 à 1920), Québec, Presses de l'Université Laval, 1998, p. 981-983. http://www.biographi.ca.

HEPBURN, Sharon, "Crossing the Border from Slavery to Freedom: The Building of a Community at Buxton, Upper Canada ", American Nineteenth Century History, 3, 2 (2002), p. 25-68.

HOULD, Fernand J., L'Institut canadien de Québec (1848-1898): agent de promotion de la cie culturelle à Québec: mythe et réalité, M.A. (Histoire), Université Laval, 1998, 168 p.

HUDON, François, avec la collaboration de Karine FORTIN et Alex HARPER, L'bistoire du Club Saint-James de Montréal (18571999), Montréal, Publications Anchor-Harper, 2000, 112 p.

HUDON, Jean-Paul, «Henri-Raymond Casgrain, Gédéon Ouimet et les livres donnés en prix dans les écoles de 1878 à 1886 », Voix et images, 26, 3 (printemps 2001), p. 596-616.

JAUMLAIN, Serge, "Le Canada" des récits de voyages et brochures de propagande diffusés en Belgique (1867-1914)", dans Serge Jaumain (dir.), Les immigrants préférés : les Belges, Ottawa, Les Presses de l'Université d'Ottawa, 1999, p. 75-100.

JONES, Preston, "Quebec and Louisiana: The Civil War Years ", Québec Studies, 23 (printemps 1997), p. 73-81.

JONES, Preston, "Civil War, Culture War: French Quebec and the American War between the States ", The Catholic Historical Rerien', 87, 1 (janvier 2001), p. 55-70.

JOYAL, Renée, «L'Acte concernant les écoles d'industrie (1869). Une mesure de prophylaxie sociale dans un Québec en voie d'urbanisation ", dans Renée Joyal (dir.), L'érolution de la protection de l'enfance au Québec : des origines à nos jours, Sainte-Foy, Les Presses de l'Université du Québec, 2000, p. 35-48.

KENNY, Stephen, «A Prejudice That Rarely Utters Its Name : A Historiographical and Historical Reflection upon North American Anti-Catholicism ", American Retien' of Canadian Studies, 32, 4 (2002), p. 639-672. 
LACROIX, Laurier, « Run Krieghoff cours », The Journal of Canadian Art History/Annales d'histoire de l'art canadien, 24 (2003), p. 60-71.

LAFLAMIME, Jean. Le théatre francophone à Montréal, de 1855 à 1880 : une institution qui tarde, Ph.D. (Histoire), Université de Montréal, 2000, 329 p.

LAMLARRE, Jean, «La participation des Canadiens français à la Guerre civile américaine (1861-1865) », Légion, (septembreoctobre 1999), p. 16-17.

LANLARRE, Jean, « De gré ou de force! L'enrôlement des Canadiens français lors de la guerre de Sécession américaine ", Légion, (septembre-octobre 2000), p. 33-36.

LAMLARRE, Jean, « Les Canadiens français et leur enrôlement dans la Guerre de Sécession américaine : une autre dimension de leur migration aux États-Unis », dans Jean Lamarre, M fartin Pâquet et Yves Frenette (dirs.), Les pariours de l'histoire : hommage à Yres Roby, Sainte-Foy, Presses de l'Université Laval, 2002, p. 263-280.

LAMARRE, Jean, «The participation of Immigrants in the American Civil War: The Case of the French Canadians ", dans Bern Horn (dir.), Forging the Nation: Perspectives on Canadian Military History, St.Catharines, Ontario, Vanwell Publishing, 2002, p. 65-80.

LAMBERT, Pierre, Les anciennes diligences du Québec : le transport en voiture publique au XIXe siècle, Sillery, Septentrion, 1998, 193 p.

LANIBERT, Pierre, "Les reillees du Père Bonsens, un journal édité à Beloeil en 1865-1866 ", Cahiers d'histoire de la Société d'histoire de BeloilMont-Saint-Hilaire, 23, 68 (2002), p. 28-37.

LAMIONDE, Yvan, " Quelle histoire racontons-nous ? Fiction littéraire et histoire ", Les Cabiers des Dix, 55 (2001), p.103-115. 
LAMONDE, Yvan, "Rome et le Vatican : la vocation catholique de l'Amérique française ou de l'Amérique anglaise ? ", dans JeanPierre Wallot (dir.), avec la collaboration de Pierre Lanthier et Hubert Watelet, Constructions identitaires et pratiques sociales : actes $d u$ colloque en hommage à Pierre Sal'ard, tenu à l'Université d'Ottawia les 4, 5, 6 octobre 2000, Ottawa, Les Presses de l'Université d'Ottawa, 2002, p. 324-343.

LANDRY, François, Beauchemin et l'édition. Une culture modèle, 18401940, Montréal, Fides, 1997, 367 p.

LEBLANC, Marcel, "Le hockey à Roberval (1875-1960)", Saguenayensia, 44, 3 (2002), p. 31-36.

LeJEUNE, Françoise, «La presse française et les rébellions canadiennes de 1837 », Rel'ue d'histoire de l'Amérique française, 56, 4 (printemps 2003), p. 481-512.

LEVASSEUR, Louis, «L'enseignement dans les collèges classiques au XXe siècle : une vision du monde en difficile harmonie avec la modemisation de la société québécoise ", Historical Studies in Education/Rer'ue d'bistoire de l'éducation, 14, 1 (printemps 2002), p. 35 66.

LITTLE, J.I., " "Labouring in a Great Cause" : Marcus Child as Pioneer School Inspector in Lower Canada's Eastern Townships, 1852-1859 ", Historical Studies in Education/Rerue d'histoire de l'éducation, 10, 1-2 (printemps-automne 1998), p. 85-115.

LOUGHEED, Richard, The Controversial Coniersion of Charles Chiniquy, Ph.D. (Théologie), Université de Montréal, 1993, 436 p.

MLARTEL, Narcel, Le deuil d'un pays imaginé. Rèies, luttes et déroute du Canada français : les relations entre le Québec et la francophonie canadienne (1867-1975), Ottawa, Les Presses de l'Université d'Ottawa, coll. "Amérique française ", $\mathrm{n}^{\circ}$ 5, 1997, $203 \mathrm{p}$.

MARTEL, Marcel, Le Canada français : récit de sa formulation et de son éclatement (1850-1967), Ottawa, La Société historique du Canada, coll. "Les groupes ethniques du Canada », $\mathrm{n}^{\circ} 24,1998,35 \mathrm{p}$. 
MLAR'TIN, Ged, «Le Rapport Durham et les origines du gouvernement responsable au Canada ", Bulletin d'bistoire politique, 6, 3 (printemps-été 1998), p. 33-51.

MONTREUIL, Sophie, «Bibliographie internationale de l'histoire de la lecture ", dans Yvan Lamonde et Sophie Montreuil (dirs), Lire au Québec an XIXe siècle, Saint-Laurent, Fides, 2003, p. 285306.

MORAN, James E., "The Ethics of Farming-Out: Ideology, the State and the Asylum in Nineteenth-Century Quebec », Bulletin canadien d'histoire de la médecine/Canadian Bulletin of Medical History, 15, 2 (1998), p. 297-316.

MORAN, James E., Committed to the State Asylum: Insanity and Society in Nineteenth-Century Quebec and Ontario, Montréal, McGillQueen's University Press, 2000, $226 \mathrm{p}$.

MUNRO, Kenneth, "Sir Charles Boucher de Boucherville », Dictionnaire biographique du Canada, XIV (de 1911 à 1920), Québec, Presses de l'Université Laval, 1998, p. 113-117.

http://www.biographi.ca.

MURRAY, Jocelyne, La scolarisation au Québec (1850-1900), l'exemple de la Mauricie, Ph.D. (Études québécoises), Université du Québec à Montréal, 1999, 498 p.

MURRAY, Jocelyne, « La scolarisation élémentaire en Mauricie (1850-1900) : esquisse de la population scolaire et des résultats de ses apprentissages ", Rerue d'histoire de l'Amérique française, 55, 4 (2002), p. 573-601.

MURRAY, Jocelyne, Apprendre à lire et à compter. École et société en Mauricie (1850-1900), Sillery, Septentrion, 2003, 278 p.

MURRAY, Kathleen Rochefort, « Sainte Anne as a Symbol of Literacy in Quebec Culture ", Québec Studies, 30 (2000-2001), p. 7078. 
MURRAY, Stuart, " Canadian Participation and National Representation at the 1851 London Great Exhibition and the 1855 Paris Exposition Universelle », Histoire sociale/Social History, 32, (mai 1999), p. 1-22.

NELSON, Wendie, " "Rage against the Dying of the Light": Interpreting the Guerre des Éteignoirs », The Canadian Historical Rerien, 81, 4 (décembre 2000), p. 551-581.

NOLLEAU, Olivier, L'encadrement religieux de la population de 1767 à 1867, M.A. (Histoire et sciences humaines), Université de Sherbrooke, 2000, $126 \mathrm{p}$.

NORCLIFFE, Glen, Ride to Modernity. The Bicycle in Canada, 1869. 1900, Toronto, University of Toronto Press, 2001, 248 p.

PAPINEAU, Lactance, Journal d'un étudiant en médecine à Paris, texte établi avec présentation et notes par Georges Aubin et Renée Blanchet, Montréal, Varia, coll. «Documents et Biographies », 2003, 256 p.

PAQUIN, Stéphane-G., "Les imaginaires nationaux et la théorie du pacte de 1867 : l'invention d'un mythe ", Bulletin d'bistoire politique, 7, 3 (printemps 1999), p. 122-137.

PARENT, Étienne, Discours, édition critique par Claude Couture et Yvan Lamonde, Montréal, Presses de l'Université de Montréal, coll. «Bibliothèque du Nouveau monde », 2000, 466 p.

PERIN, Roberto, "Ultramontanisme et modernité : l'exemple d'Alphonse Villeneuve (1871-1891) ", dans Yves Frenette (dir.), Les pariours de l'histoire : hommage à Yies Roby, Sainte-Foy, Les Presses de l'Université Laval, 2002, p. 305-325.

PETRIE, Brian M., "The French-Canadian Patriote Convict Experience (1840-1848) ", Journal of the Royal Australian Historical Society, 81, 2 (1995), p. 167-183.

POITRAS, Claire, «Tertiarisation et transformation de l'espace urbain : la rue NcGill à Montréal (1842-1934) ", Urban History Reicew/Relue d'histoire urbaine, 31, 2 (2003), p. 3-17. 
POTIER-BRIDAY, David, L'influence du clergé catholique sur les comportements électoraux dans les comtés de Saint-Hyacinthe et de Bagot lors des élections de 1867, M.A. (Histoire), Université du Québec à Montréal, 1999, 208 p.

POUTANEN, Mary Anne, "Unless she gives better satisfaction": Teachers, Protestant Education, and Community in Rural Quebec, Lochaber and Gore District, 1863-1945 ", Historical Studies in Education/Rerue d'bistoire de l'éducation, 15, 2, (automne 2003), p. 237-272.

RACINE, Jacques, "Citoyen de Québec, citoyen du monde : Stanislas Lortie (1869-1912) ", dans Gilles Routhier et JeanPhilippe Warren (dirs.), Les irsages de la foi : figures marquantes du catholicisme québécois, Montréal, Fides, 2003, p. 147-159.

RAJOTTE, Pierre (dir.), Lieux et réseaux de sociabilité littéraire au Québec, Préface de Alain Viala, Québec, Éditions Nota bene, coll. "Séminaires », 2001, 335 p.

RAMIIREZ, Bruno, La ruée vers le sud: migrations du Canada iers les États-Unis, 1840-1930, Montréal, Boréal, 2003, 276 p.

RÉNILLARD, Marie-Paule, La construition d'une polémique : l'annexionnisme dans deux journaux montréalais L'avenir et The Montreal Gazette (1849-1850), M.A. (Études françaises), Université de Montréal, 1997, 225 p.

ROBERT, Jean-Claude, «Réseau routier et développement urbain dans l'île de Montréal au XIXe siècle ", dans Horacio Capel et Paul-André Linteau (dirs.), Barielona-Montréal. Desarollo urbano comparado/Déeloppement urbain comparé, Barcelone, Publications de la Universitat de Barcelona, 1998, p. 99-115.

ROMNEY, Paul, " Provincial Equality, Special Status and the Compact Theory of Canadian Confederation ", Canadian Journal of Political Science/Rerue canadienne de science politique, 32, 1 (mars 1999), p. 21-39. 
ROUSSEAU, Louis, " Peut-on parler d'une histoire religieuse quantitative au Québec ", dans L'observation quantitative du fait religieux, colloque de l'Association française d'histoire religieuse contemporaine, Lille, Centre d'histoire de la région Nord et de l'Europe du Nord-Ouest de l'Université Charles-de-Gaulle-Lille III, 1992, p. 73-90.

ROY, Jean, «La fabrique, les couvents et les académies dans le diocèse de Nicolet (1852-1904) ", Annales de Bretagne et des Pays de l'Ouest, 110, 4 (2003), p. 95-106.

RUDIN, Ronald, Founding Fathers: The Celebration of Champlain and Laial in the Streets of Quebec, 1878-1908, Toronto, University of Toronto Press, 2003, xi-290 p.

SABOURIN, Hélène, « P.-J.-O. Chauveau et les débuts de la Chambre des arts et manufactures du Bas-Canada 1857-1872», Scientia Canadensis, XVI, 2 (1992), p. 128-153.

S.ABOURIN, Hélène, P.-J.-O. Chameau et l'éducation 1855-1873: une relecture, Ph.D. (Histoire), Université du Québec à Montréal, 2001, $\mathrm{x}-324 \mathrm{p}$.

S.ABOURIN, Hélène, À l'école de P.-J.-O. Chaureau : Éducation et culture au XIXe siècle, Montréal, Leméac, 2003, 230 p.

SANFILIPPO, Matteo, «Du Québec à Rome et de Rome au Québec : voyageurs canadiens-français en Italie et voyageurs italiens au Canada français entre la deuxième moitié du XIXe siècle et le début du XXe », dans Jean-Pierre Wallot (dir.), avec la collaboration de Pierre Lanthier et Hubert Watelet, Constructions identitaires et pratiques sociales, Ottawa, Les Presses de l'Université d'Ottawa, 2002, p. 279-301.

SAVARD, Pierre, "Stanislas-Alfred Lortie ", Dictionnaire biographique du Canada, XIV (de 1911 à 1920), Québec, Presses de l'Université Laval, 1998, p. 720-722. p. http://www.biographi.ca.

SÉGUIN, Renaud, Un loyalisme incendiaire : étude sur le "torysme » montréalais de 1849 à partir de journaux de l'époque, M.A. (Histoire), Université de Montréal, 2002, vii-172 p. 
SICO'T"TE, Hélène, « Le rôle de la vente publique dans l'essor du commerce d'art à Montréal au XIXe siècle - Le cas de W. Scott \& Sons ou comment le marchand d'art supplanta l'encanteur ", The Journal of Canadian Art History/Annales d'histoire de l'art canadien, 23, 1-2 (2002), p. 6-33.

SKIDMORE, Colleen, "Triumphal Arches at Montreal, 1860 ", Journal of Canadian Art History/Annales d'histoire de l'art canadien, 19, 1 (1998), p. 86-109.

SKIDMORE, Colleen, "Photography in the Convent: Grey Nuns, Quebec, 1861 », Social History/Histoire sociale, 35, 70 (2002), p. $279-310$.

THÉRIO, Adrien, Joseph Guibord : victime expiatoire de l'érêque Bourget. L'Institut canadien et l'affaire Guibord reirisités, Montréal, XYZ, 2000, $270 \mathrm{p}$.

TOCQUEVILLE, Alexis de, Regards sur le Bas-Canada, Choix de textes et présentation de Claude Corbo, Montréal, Typo, 2003, $336 \mathrm{p}$.

VIGNEAULT, Michel, La renaissance d'un sport organisé au Canada : le bockey à Montréal (1875-1917), Ph.D. (Histoire), Université Laval, 2001, 2 volumes.

VOISINE, Nive, «D’Église et d'université : Louis-Adolphe Pâquet (1859-1942) ", dans Gilles Routhier et Jean-Philippe Warren (dir.), Les iisages de la foi : figures marquantes du catholicisme québécois, Montréal, Fides, 2003, p. 123-132.

WATKINS, Meredith G., "The Cemetery and Cultural Memory: Montreal (1860-1900) », Urban History Retien/Rerue d'bistoire urbaine, 31, 1 (automne 2002), p. 52-62.

\section{Les idées, la ville et l'usine (1880-1929)}

ADAMS, Annmarie et Peter GOSSAGE, « Chez Fadette: Girlhood, Family, and Private Space in Late Nineteenth-Century Saint-Hyacinthe ", Urban History Reriew/Rerue d'bistoire urbaine, 26, 2 (mars 1998), p. 56-68. 